\title{
Développements récents dans le domaine du micro-usinage par lasers excimères
}

\author{
E. Miquet \\ IREPA LASER, Parc d'Innovation, 67400 Illkirch, France
}

\begin{abstract}
Résumé : Pour répondre aux besoins particuliers des industries de l'électronique et de l'instrumentation médicale, l'IREPA LASER s'est équipé depuis trois ans d'un centre d'usinage constitué d'un laser excimère et d'un ensemble mécano-optique. Le propos de cet exposé est de présenter deux applications concernant l'industrie horlogère et l'industrie d'instrumentation médicale ayant fait l'objet d'un transfert industriel.
\end{abstract}

Les lasers excimères, découverts dès 1975, ont enregistré ces dernières années de profonds développements technologiques. Ils constituent aujourd'hui la 3ème catégorie des lasers industriels après les lasers $\mathrm{CO} 2$ et $\mathrm{YAG}$.

Les lasers excimères sont des sources U.V. impulsionnelles présentant une faible dispersion spectrale (quelques dixièmes de nanomètres). La longueur d'onde émise dépend du type de mélange de gaz présent au sein de la cavité. Parmi ceux-ci, ArF (193 nm), KrF (248 nm), XeCl (308 nm) et XeF ( $351 \mathrm{~nm})$ sont les plus utilisés. Les générations actuelles de lasers excimères délivrent une puissance moyenne inférieure à $200 \mathrm{~W}$, un taux de répétition maximum de $1 \mathrm{kHz}$, une énergie comprise entre quelques $\mu \mathrm{J}$ à quelques Joules et une durée d'impulsion qui peut varier entre 10 et $250 \mathrm{nsec}$.

Toutes ces caractéristiques ont permis le développement de nombreuses applications dans des domaines aussi différents que l'électronique, la médecine et la spectroscopie.

Très récemment, au début des années 80 , l'industrie de la micro-électronique s'est beaucoup intéressée aux lasers U.V. comme source de rayonnement de courte longueur d'onde susceptible de remplacer les lampes à xénon ou à mercure pour l'irradiation des résines photosensibles.

Plusieurs chercheurs ont vu alors la possibilité d'utiliser le rayonnement ultraviolet de très courte longueur d'onde ( 180 à $260 \mathrm{~nm}$ ) pour réaliser de la gravure directe (sans révélation en phase humide) à la surface de plusieurs polymères. C'est grâce aux travaux de Srinivasan (IBM) que la photoablation des polymères est véritablement née.

Pour répondre aux besoins particuliers des industries de l'électronique et de l'instrumentation médicale, l'IREPA LASER s'est équipé depuis trois ans d'un centre d'usinage constitué d'un laser excimère (LUMONICS EX 748) et d'un ensemble mécano-optique (OPTEC SA). Celui-ci comprend une table X-Y (course $250 \mathrm{~mm} \times 250 \mathrm{~mm}$ ) pilotée par une commande numérique et un projecteur optique permettant la reproduction de l'image d'un masque sur un échantillon avec un taux de réduction variable compris entre 1 et 15 .

Notons que ce centre d'usinage peut également être associé à un laser CO2 TEA, et prochainement à un laser YAG déclenché $(1064 \mathrm{~nm}, 532 \mathrm{~nm}, 353 \mathrm{~nm}$ et $266 \mathrm{~nm})$ par simple échange des miroirs et des lentilles du projecteur optique.

Le propos de cet exposé est de présenter deux applications ayant fait l'objet d'un transfert industriel.

Lâ première de ces applications conceme l'industrie horlogère.

Face à la concurrence de l'Extrême Orient, l'industrie horlogère doit concentrer ses efforts sur les nouvelles fonctions à haute technicité.

Au terme d'une étude de deux ans, une PMI franc-comtoise lance sur le marché mondial une génération de montres solaires. En lieu et place du cadran, une cellule solaire capte l'énergie utilisée par le mouvement qui fonctionne dès l'instant où il est exposé à la lumière. 
Conçu pour fonctionner dix ans et plus, le mouvement solaire possède, grâce au stockage des surplus d'énergie dans un super condensateur situé à la place de la pile, une réserve de marche d'environ 190 heures dans l'obscurité complète.

La cellule solaire est constituée d'un empilement de couches minces ( $\mathrm{SnO} 2$ - Silicium amorphe et Aluminium) déposées sur un substrat en verre. L'épaisseur cumulée des trois couches avoisine le micron.

Le département micro-usinage de l'IREPA LASER a mis au point une technique d'enlèvement de ces couches par laser Excimère sans endommager le support en verre, les critères esthétiques étant très sévères sur ce point, et sans altérer la fonctionnalité électrique de la montre. Le petit guichet à travers lequel apparaît la date est le résultat de cette application.

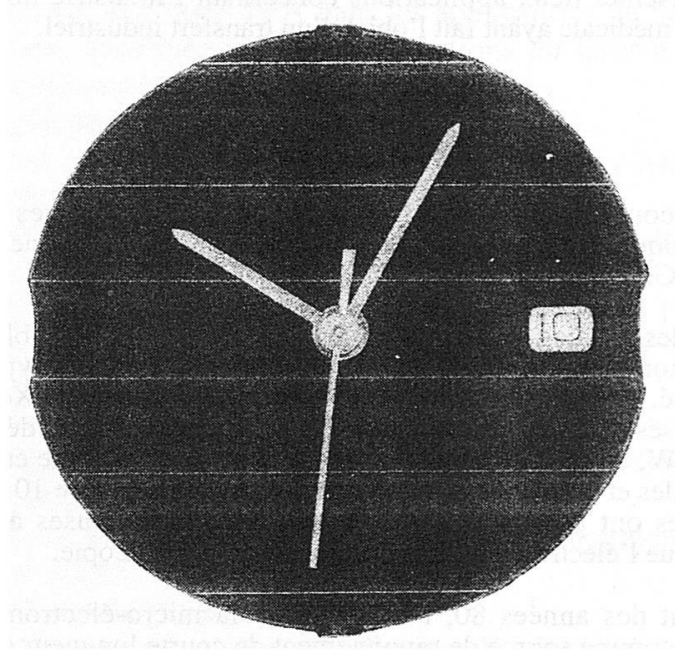

Figure 1 : Le petit guichet est le résultat de cette application

L'industrialisation du procédé a consisté à usiner les cellules par plaques de grandes dimensions (chaque plaque représente plus d'une centaine de cellules) en utilisant un masque de contact. L'utilisation d'un montage garantissant la précision des positionnements des plaques à usiner et du masque de contact grâce à des butées fixes et un maintien des plaques par aspiration est indispensable. Aussi le choix du matériau du masque de contact est déterminant pour assurer la transparence du verre après traitement au faisceau laser.

La deuxième application concerne l'industrie de l'instrumentation médicale. Il s'agit de membranes en matériau polymère bio-compatible qui serviront aux soins des grands brûlés. La membrane a une épaisseur de $30 \mu \mathrm{m}$ et se présente sous forme de feuilles de $100 \mathrm{~mm} \times 100 \mathrm{~mm}$. Le cahier des charges de ce produit stipule que doivent être percés 151.200 trous de diamètre $60 \mu \mathrm{m} \pm 20 \mu \mathrm{m}$ et 120 trous de diamètre $500 \mu \mathrm{m} \pm 20 \mu \mathrm{m}$ par feuille. La position des trous doit être précise à $\pm 20 \mu \mathrm{m}$. D'autre part il faut apporter un soin particulier lors de la manipulation des membranes en raison des risques de déchirements. 


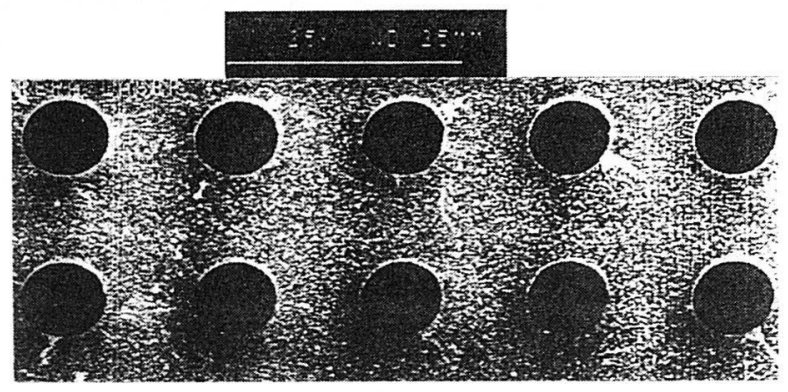

Figure 2 : Perçage de membranes en matériaux polymère

L'industrialisation du procédé a conduit à utiliser deux masques : un masque pour percer les petits trous et un masque pour percer les gros trous. La première opération consiste à percer les petits trous : 240 petits trous sont réalisés à la fois ( 240 trous sont reproduits 70 fois sur une ligne elle même reproduite 9 fois). Ensuite, après changement automatique du masque, les gros trous sont percés, un par un. Notons également que les membranes sont usinées deux par deux (empilage). Lors de l'usinage, le maintien de la membrane est assuré par soufflage d'air à faible pression.

\section{Remerciements}

L'auteur remercie A. Biernaux (Lumonics) 